\title{
Intra-arterial 5-bromo-2-deoxyuridine (BUdR) radiosensitization with external beam radiation in rhesus monkeys: A toxicity study
}

Harry S. Greenberg ${ }^{3}$, William F. Chandler ${ }^{6}$, Richard F. Diaz ${ }^{4}$, Damon R. Averill ${ }^{2}$, Stephen S. Gebarski ${ }^{5}$, Allen S. Lichter ${ }^{4}$ and William D. Ensminger ${ }^{1}$

Departments of Internal Medicine ${ }^{1}$, Laboratory Animal Medicine ${ }^{2}$, Neurology $^{3}$, Radiation Oncology ${ }^{4}$, Radiology ${ }^{5}$, and Surgery, Division of Neurosurgery ${ }^{6}$, University of Michigan Hospitals, Ann Arbor, Michigan, USA

Key words: 5-bromo-2-deoxyuridine (BUdR), malignant astrocytomas, monkeys, radiation, radiosensitization

\section{Summary}

A primate toxicity study was performed to test the hypothesis that BUdR does not increase the likelihood of unilateral or bilateral central nervous system damage secondary to radiation therapy.

BUdR, a halogenated pyrimidine analog is incorporated into DNA of dividing cells and sensitizes them to radiation. It is best given unilaterally, intra-arterially by continuous infusion because of its regional advantage $\left(R_{d}\right)$ estimated to be between 11 and 16.

Six rhesus monkeys were implanted with a Model 400 Infusaid pump perfusing the right internal carotid artery. Three of the six monkeys received intra-arterial (IA) BUdR infusion plus whole brain external beam radiation (6000 $R$ over 6 weeks) and three received radiation alone.

The two BUdR treated animals completing radiation developed symmetric bilateral high signal aberrations on MRI in the frontal, parietal, and occipital centrum semiovale and corona radiata at nine months. At autopsy, confluent microinfarcts were found to correspond topographically to the MRI abnormalities. In the radiation alone group, two animals had normal MRI and autopsy while the third animal had bilateral MRI high signal aberrations develop sequentially with corresponding microinfarcts at autopsy. These changes were greater in severity than those seen in the BUdR treated animals. We support previous evidence that there is differential intraspecies sensitivity to radiation. We find that BUdR produces no unilateral potentiation of radiation toxicity.

\section{Introduction}

Radiation therapy has been shown to be the most effective post-surgical single modality treatment for malignant astrocytomas, and therapeutic responses are dose-dependent $[1,2]$. BUdR is a halogenated pyrimidine analog that is incorporated into DNA of dividing cells in a competitive manner with thymidine and sensitizes them to radiation therapy [3,4]. It is given unilaterally, intra-arterially by continuous infusion because of its $R_{d}$ estimated to be between 11 and 16 [5]. Therefore the hemisphere ipsilateral to the infusion will receive $11-16$ times greater BUdR concentration than the opposite hemisphere. We performed a primate toxicity study to test the hypothesis that BUdR does not increase the likelihood of unilateral or bilateral damage secondary to radiation therapy.

The brain appears to be an ideal site for BUdR radio-sensitization, since neurons, glia and endothe- 
lial cells have a very low mitotic rate, will not take up BUdR and, hence, will not be sensitized. Malignant astrocytomas will incorporate BUdR and be radiosensitized. A therapeutic advantage should be obtained between tumor and normal brain. Prior to beginning a human study of IA BUdR radiosensitization in patients with malignant astrocytomas we implanted 6 rhesus monkeys with a Model 400 Infusaid pump perfusing the right internal carotid artery to investigate normal brain toxicity. Three monkeys received IA BUdR infusion. All monkeys received whole brain external beam radiation.

\section{Methods}

Six normal rhesus monkeys had a Model 400 Infusaid pump implanted in a subcutaneous pocket on the right side of the abdomen with the drug outlet cathether tunneled subcutaneously and advanced retrograde down the right external carotid artery and secured so that the orifice of the cathether was at the carotid artery bifurcation [6]. All distal branches of the external carotid artery were ligated so that a purely internal carotid artery infusion was achieved. Animals received a 5-day postoperative course of antibiotics. At the time of implantation each pump was filled with heparinized saline and refilled at bimonthly intervals until optimal wound healing. After one month, 3 monkeys (\#1-3) had the pump filled with BUdR $10 \mathrm{mg} / \mathrm{kg} /$ day (in a carbonatebicarbonate buffer, pH 9.7) containing a 1000 units/ $\mathrm{ml}$ of heparin. The control group ( $\$ 4-6)$ received the same carbonate-bicarbonate buffer solution containing a 1000 units $/ \mathrm{ml}$ of heparin. The day following pump fill, all animals began cobalt 60 external beam radiation, which was administered four days per week using opposed lateral whole brain treatment portals with $250 \mathrm{cGy}$ (rad) midplane dose being given daily under general anesthesia with Ketamine and Rompun. Total dose was $6000 \mathrm{cGy}$ (rad) over 40 elapsed days. Shielding was utilized to protect the orbit and pharynx regions. Following completion of the radiation all pumps were explanted.

MRI was obtained before radiation (baseline) and at 3 and 9 months following completion of RT. The
9 month MRI was immediately prior to sacrifice and pathological examination. All MRIs were acquired with a super conducting 0.35 Tesla Diasonics $\mathrm{MT} / \mathrm{S}$ unit imaging hydrogen protons via spin-echo technique. Both spin-lattice relaxation time $\left(T_{1}\right)$ and spin-spin relaxation time $\left(\mathrm{T}_{2}\right)$ weighted $5 \mathrm{~mm}$ axial sections were performed to image the entire cranial contents, resulting in an effective resolution of $1.8 \mathrm{~mm}$. These used a $128 \times 256$ matrix and the standard head coil. No paramagnetic contrast was employed. Images were interpreted in a single blind fashion, qualitatively rating presence or absence of signal aberrations and their topographic distribution. As the $T_{2}$ imager provided the most signal aberrations they were quantified by assigning a scale from least $(1+)$ to most severe $(3+)$ signal aberrations.

The animals were sacrificed by administration of pentobarbital sodium IV until there was cardiorespiratory arrest. Complete necropsies were done with brain, spinal cord, and somatic tissues removed and immersion-fixed in $10 \%$ neutral buffered formalin. After 3 weeks fixation, the brain was sliced in the coronal plane into $0.5 \mathrm{~cm}$ slabs. Identical blocks were collectd from each hemisphere including prefrontral, frontal, parietal, temporal and occipital lobes. Additional bilateral blocks were collected from thalamus, hypothalamus, hippocampus, midbrain, pons, cerebellum, medulla and spinal cord. These specimens were embedded in paraffin, sectioned at $\operatorname{six} \mu \mathrm{m}$ and stained with hematoxylin-eosinluxol fast blue using standard methods. The slides were examined in a single blind fashion. In an effort to quantitate histologic lesions all the sections were examined with a dissecting microscope at $100 \times$ magnification. At this level of magnification all lesions equal to or larger than $0.1 \mathrm{~mm}$ were tabulated for each hemisphere. After all animals had been sacrificed and examined, comparison of MRI and autopsy results took place by both comparison of tabular data and direct comparison of MRI images with histologic sections.

Animal care was provided by the staff of the Unit for Laboratory Animal Medicine under the supervision of veterinarians by the guidelines provided for in 'The Guide for the Care and Use of Laboratory Animals', DHEW Publ. No. (NIH) 78-23 Revised 
1978. This included regular periodic surveillance of animal facilities, review of programs for humane use of animals, and the appropriate use of surgical anesthesia, analgesics and tranquilizers.

\section{Results}

Animal \#1 died an anesthetic death after 16 radiation treatments (Table 1). Animals \#2 and 3 (IA BUdR + radiation) had normal MRI at baseline and 3 months, but had bilateral diffuse mild periependymal and cortical-medullary junction high signal abnormalities develop on $\mathrm{T}_{2}$ weighted sequencies at 9 months. At autopsy histologic changes were limited to the frontal, parietal and occipital centrum semiovale and corona radiata. Discrete oval regions of pallor on low magnification had a variety of histologic changes. The majority of lesions were focal necrosis with astrogliosis, mineralization, loss of myelin and axons and lipid hemosiderin-laden macrophages (Fig. 1a). Rare acute lesions had hemorrhagic necroses with swollen eosinophilic axons and edema. Other pale areas had diffuse hypertrophic astrocytes (Fig. 1b). Some of these microinfarcts were associated with vascular changes with massive hyalin thickening of the vessel wall (Fig. 1c) and either endothelial loss or hypertrophy. A few small arteries were found with occluded lumens or recanalized thrombi. Perivascular gliosis was rare and occasional small perivascular cuffs of mononuclear leu- cocytes were present at the margin of larger lesions. Histologic changes were symmetrical on animal \#2. Animal \#3 had more lesions in the non-BUdRperfused hemisphere.

Animals \#4 and 6 (radiation alone) had normal MRI at baseline, 3 and 9 months, as well as a normal autopsy. Animal \#5 (radiation alone) had symmetric high signal abnormalities on $\mathrm{T}_{2}$ weighted sequences develop between baseline and 3 months with these signal aberrations increasing in severity at 9 months (Fig. 2). Autopsy showed bilateral symmetrical microinfarcts which were greater in number than in our BUdR + radiation animals (Fig. 3). Correspondence of autopsy and MRI data was good with the most marked histologic lesions exactly matching the topography of the most marked MRI signal aberrations. In no animal was an MRI signal aberration identified that did not correspond to a confluence of the above described histologic lesions. In no animal was a confluence of these lesions found on autopsy without a corresponding MRI signal aberration.

\section{Discussion}

Monkeys were chosen as the experimental animal because they are similar to man and have an internal carotid artery dominant supratentorial brain perfusion. The radiation dosage schedule was similar to the one to be used in our patients with malignant astrocy-

Table 1. IA BUdR Radiosensitization + radiation therapy (RT) ${ }^{\mathrm{a}}$

\begin{tabular}{|c|c|c|c|c|c|}
\hline \multirow[t]{2}{*}{ Treatment } & \multirow[t]{2}{*}{ Animal } & \multirow[t]{2}{*}{$\begin{array}{l}\text { MRI Signal } \\
3 \text { months }\end{array}$} & \multirow[t]{2}{*}{$\begin{array}{l}\text { Aberrations } \\
9 \text { months }\end{array}$} & \multicolumn{2}{|c|}{$\begin{array}{l}\text { Histopathology } \\
\text { (number of micro- } \\
\text { infarcts } 0.1 \mathrm{~mm} \text { ) }\end{array}$} \\
\hline & & & & (R) & (L) \\
\hline $\mathrm{BUdR}^{\mathrm{b}}+\mathrm{RT}$ & 1 & Anesthetic death & & - & - \\
\hline$B U d R^{b}+R T$ & 2 & Normal & 1+ Mild L,R Frontoparietal & 2 & 4 \\
\hline $\mathrm{BUdR}^{\mathrm{b}}+\mathrm{RT}$ & 3 & Normal & $2+$ Moderate L, $\mathrm{R}$ & 3 & 11 \\
\hline RT & 4 & Normal & Normal & 0 & 0 \\
\hline RT & 5 & $1+$ Mild Symmetric & $3+$ Severe Symmetric & 40 & 31 \\
\hline RT & 6 & Normal & Normal & 0 & 0 \\
\hline
\end{tabular}

a Radiation therapy - $6000 \mathrm{cG} @ 250 \mathrm{cGy} /$ day 4 days/week $\times 6$ weeks.

${ }^{\mathrm{b}}$ BUdR-(R) internal carotid system $(10 \mathrm{mg} / \mathrm{kg} /$ day $\times 6$ weeks $)$ via implanted Infusaid ${ }^{(\mathrm{R})}$ pump. 

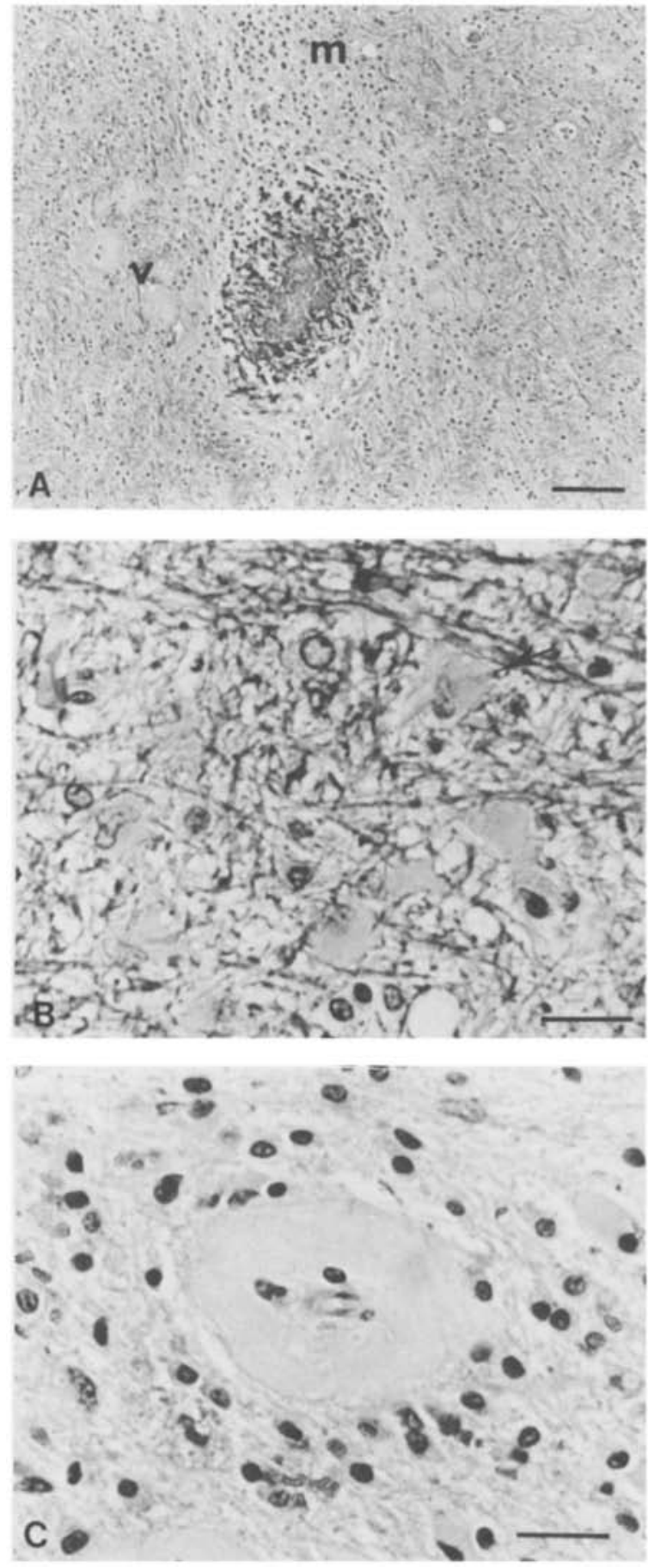

Fig. I. Monkey \#3. a. Right occipital white matter. A typical mineralized microinfarct with adjacent hyalinized small vessels (V) and hemosiderin-laden macrophages (M). Horizontal bar $0.5 \mathrm{~mm}$ H-E-luxol fast blue. b. Frontal white matter - hypertrophic astrocytes (arrow). Horizontal bar $0.25 \mathrm{~mm} \mathrm{H}$-E-luxol fast blue. c. Adjacent to $2 \mathrm{a}$ - hyalin thickening of small vessel with attenuated endothelium lined lumen. Horizontal bar $0.25 \mathrm{~mm} \mathrm{H}$-E-luxol fast blue. tomas. BUdR was given continuously throughout radiation therapy to stimulate planned clinical studies in patients with malignant gliomas. Greater exposure to BUdR would allow maximum incorporation into the DNA of dividing tumor cells. BUdR dose was selected to achieve plasma levels comparable to that in our planned clinical study.

The histology of the cerebral white matter lesions in our primates are similar to those described by Caveness in animals treated with doses of radiation conventionally given in whole brain human radiation (7). In our animals, identically treated monkeys showed considerably variation in brain injury. Of three animals receiving radiation alone, two $(\# 4,6)$ had normal MRI and autopsies while a third (\#5) demonstrated sequential development of the most severe MRI $\left(\mathrm{T}_{2}\right)$ high signal abnormalities. We add to existing evidence that a differential intraspecies sensitivity to radiation exists.

The MRI signal aberrations in animal \#5 as well as similar but less severe abnormalities in \#2 and 3 showed topographic and grade correspondence with white matter microinfarcts at postmortem examination. Animals \#2 and 3 developed bilateral MRI signal aberrations at 9 months while animal \#5 developed sequential changes at 3 and 9 months. These lesions were symmetrical in animal $\# 2$ and more numerous in the non-BUdR perfused hemisphere of animal \#3. In one animal we were able to image with MRI the sequential changes of radiation toxicity. The biologic basis for the MRI signal aberrations is reported to be secondary to the expected prolongation of $T_{2}$ from regional increase in water content as seen in edema and gliomas [8].

Intra-arterial BUdR produced no additional toxicity ipsilateral to the arterial infusion in the hemisphere receiving 11-16 times higher concentration of BUdR. However, of two BUdR animals both had bilateral MRI and postmortem changes compared to one of three animals in the control groups. The changes in the control animal were most severe. Whether the more frequent bilateral toxicity in the BUdR treated animals was secondary to IA BUdR, where we would expect it to be greater in the ipsilateral hemisphere and where in one animal \#3, it was greater in the contralateral hemisphere or to differential intraspecies sensisitivity to radiation is uncertain. 

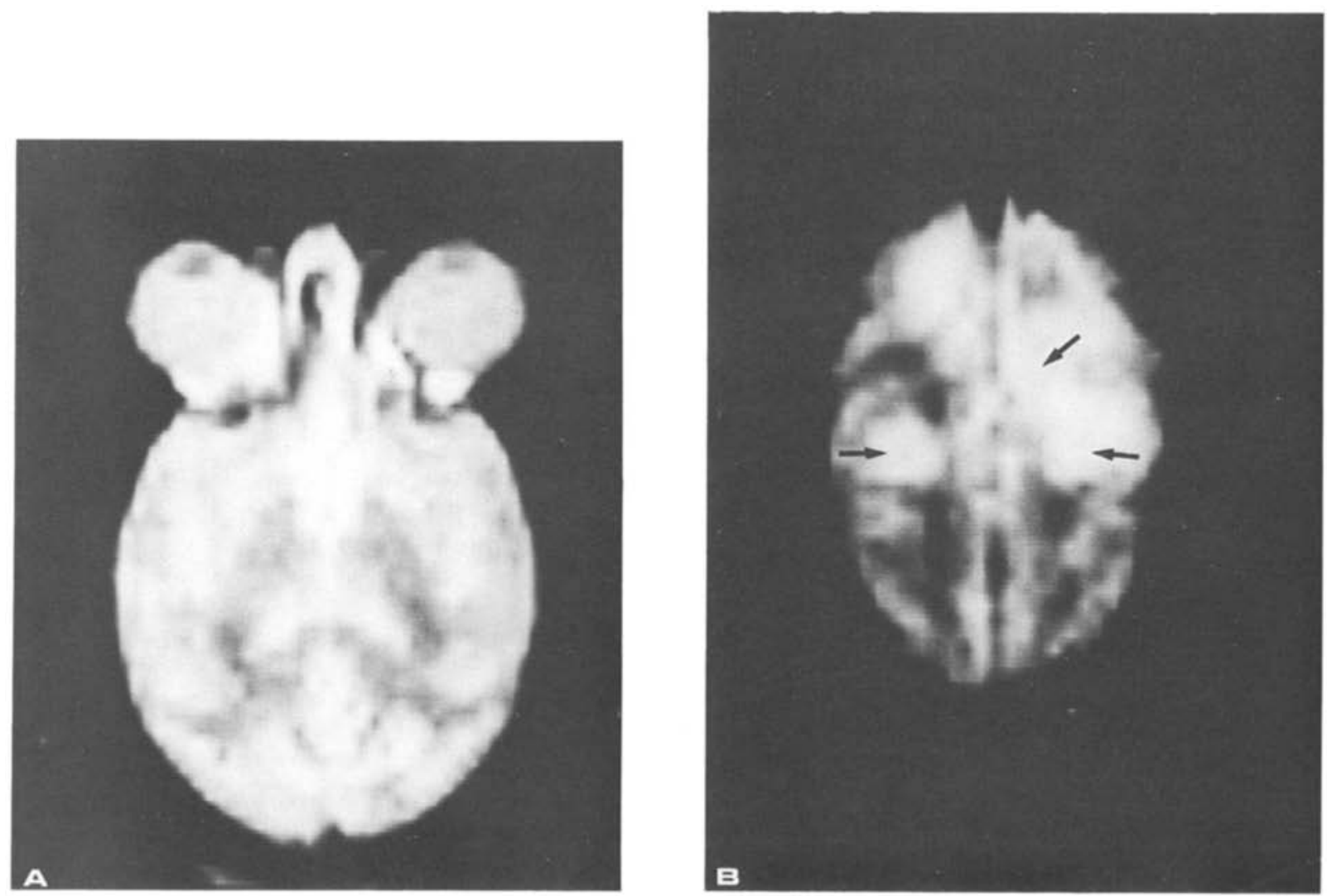

Fig. 2. MRI, monkey \#5, nine months. Severe diffuse, symmetric predominantly white matter signal aberrations. (Figure $2 b$ ). These are most marked in the centrum semiovale (arrows).

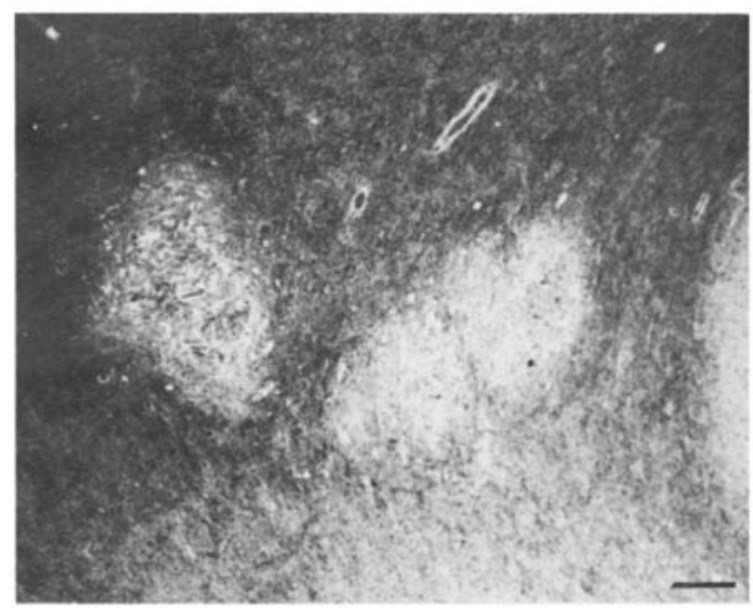

Fig. 3. Monkey \#5. Left parietal centrum semiovale. Three necrotic microinfarcts, the left most containing axonal mineralization. Horizontal bar $1.0 \mathrm{~mm}$ H-E-luxol fast blue.
We believe this is a safe model for the future study of regionally applied halopyrimidines. Hoshino et al. used percutaneous BUdR carotid infusion in Japan with a high frequency of cerebral vascular complications $[9,10]$. Our pump system is implanted surgically retrograde down the external carotid artery. The catheter does not protrude into the internal carotid artery, and heparin is placed in the pump to prevent thromboembolic complications. The intravenous infusion of BUdR has been accompanied by systemic toxicity including diffuse skin rash and hematologic toxicity $[11,12]$. In recent studies by Hoshino et al. with a BUdR monoclonal antibody they have found an $\mathrm{S}$ phase labelling in glioblastoma multiforme varying between 1.3 and $27 \%$ [13]. This indicates that a prolonged constant infusion may be necessary for BUdR to be incorporated into and sensitize all cells. We hope $R_{d}$ of BUdR will enable us to use a lower radiation dose with increase thera- 
peutic effect and less systemic toxicity.

We have begun a Phase I clinical trial (NCI Protocol T-83-101) in patients with Grade III-IV malignant astrocytomas. This employs Infusaid pump placement for eight weeks IA carotid BUdR infusion [6]. Focal external beam radiation therapy is delivered the last six weeks of BUdR infusion.

\section{Acknowledgements}

This work was partially supported by NIH grant number 1 R01 CA33768-01A3 and research grant RR 00200-21 from the Shiley Infusaid Corporation. The Upjohn Center for Clinical Pharmacology was used.

\section{References}

1. Walker MD, Alexander $\mathrm{J}_{\Gamma}$ E, Hunt WE, MacCarthy CS, Mahaley J, Norrell HA, Owens G, Ranshoff J, Wilson DB, Gehan EA, Strike TA: Evaluation of BCNU and/or radiotherapy in the treatment of anaplastic gliomas: A cooperative clinical trial. J Neurosurg 49:333-343, 1978

2. Walker MD, Strike TA, Sheline GE: An analysis of doseeffect relationship in the radiotherapy of malignant gliomas. Int J Radiat Oncol Biol Phys 5:1725-1733, 1979

3. BUdR: Clinical brochure, IND 2197 NSC 38297 , July 1980

4. Djordjevic, Szybalski W: Genetics of human cell lines. III. Incorporation of 5-bromo and 5-iododeoxyuridine into the deoxyribonucleic acid of human cells and its effect on radiation sensitivity. J Exptl Med 112:509-531, 1960

5. Russo A, Gianni L, Kinsella TJ, Klecker Jr RW, Jenkins J, Rowland J, Glatstein E, Mitchell JB, Collins J, Meyers C: A pharmacological evaluation of intravenous delivery of 5-bromodeoxyuridine to patients with brain tumors. Cancer Research 44:1702-1705, 1984
6. Phillips TW, Chandler WF, Ensminger WD, Greenberg HS, Seeger JF, Doan K, Gyves JW: A new implantable continuous intracarotid drug delivery system for the treatment of malignant gliomas. Neurosurgery 11:213-218, 1982

7. Caveness WF: Experimental observations: Delayed necrosis in normal monkey brain. In: Gilbert EA and Kager AR (eds) Radiation Damage to the Nervous System. New York, Raven Press, 1980, pp 1-38

8. Unger EC, Gado MH, Fullirg KF, Littlefield JL: Acute Cerebral Infarction in Monkeys: An Experimental Study Using MR Imaging. Radiology 162:789-795, 1987

9. Hoshino T: Radiosensitization of brain tumors. In: Deely TF (ed) Modern Radiotherapy and Oncology - Central Nervous System Tumors. London, Butterworths, 1974, pp $170-184$

10. Sano K, Nagai M, Arai T, Hoshino T: Follow-up results of BAR therapy of malignant brain tumors. In: Fusek I and Kunc Z (eds) Proceedings of the Fourth European Coingress of Neurosurgery, Present Limits of Neurosurgery. Amsterdam, Excerpta Medica, 1972, pp 71-75

11. Kinsella TJ, Mitchell JB, Russo A, Aiken M, Morstyn G, Hsu SM, Rowland J, Glatstein E: Continuous intravenous infusions of bromodeoxyuridine as a clinical radiosensitizer. J Clin Oncol 2:1144-1150, 1984

12. Kinsella TJ, Russo A, Mitchell JB, Rowland J, Jenkins J, Schwade J, Myers CE, Collins JM, Speyer J, Kornblith P, Smith B, Kufta C, Glatstein E: A phase I study of intermittent intravenous bromodeoxyuridine (BUdR) with conventional fractionated irradiation. Int J Radiation Oncol Biol Phys 10:69-76, 1984

13. Hoshino T, Nagashina T, Cho KG, Minovic JA, Hudes JE, Wilson CB, Edwards MSB, Pitts LH: S-Phase fraction of human brain tumors in situ measured by uptake of bromodeoxyuridine. Int J Cancer 38:369-374, 1986

Address for offprints: H. S. Greenberg, Department of Neurology, 1914/316 Taubman Center, University of Michigan Hospitals, Ann Arbor, MI 48109-0316, USA 